\title{
Special Issue on Perspectives on Thermal Plasma Research for Industrial Applications: Introduction
}

\author{
Anthony B. Murphy ${ }^{1}$
}

Published online: 20 March 2015

(C) Springer Science+Business Media New York 2015

Thermal plasmas have very useful properties that are not easily achievable using other means, in particular high power densities, high temperatures, high-velocity flows, high fluxes of active species and intense radiative emission. Moreover they are available in a wide range of configurations (direct-current transferred arcs, direct-current non-transferred arcs or plasma torches, alternating-current arcs, radio-frequency inductively-coupled plasmas, microwave plasmas and laser-produced plasmas) and powers (from hundreds of watts to a hundred megawatts). It is therefore not surprising that they find application in many industrial processes, including those for joining, melting, cutting, coating and deposition, refining, waste treatment, chemical analysis, lighting, propulsion and circuit interruption. It is these many applications that underlie all thermal plasma research; even when arcane questions such as the extent of deviations from local thermodynamic equilibrium in an arc are being investigated, the ultimate goal is to develop better thermal plasma processes for industry.

This special issue brings together eight articles written by leading researchers from universities, industry and government research laboratories, presenting their perspectives on particular aspects of thermal plasma research. Each author was asked to present their views on important topics for research and development, outstanding scientific questions, and barriers to future success. The aim is not to present a comprehensive overview of the subject. Rather, the intention is that the special issue will guide researchers towards the important topics that warrant their close attention, and that are critical for future progress in the field.

Three of the articles take a wider view, looking at thermal plasma sources, diagnostics and modelling respectively, across a range of applications. The first article considers plasma torches and inductively-coupled plasmas in particular, the second looks mainly at diagnostics of transferred arcs, while the third examines modelling issues that relate to

Anthony B. Murphy

Tony.Murphy@csiro.au

1 CSIRO Manufacturing Flagship, PO Box 218, Lindfield, NSW 2070, Australia 
many types of plasmas. These three papers give the reader an impression of the breadth of application of thermal plasmas, and of the many approaches used to produce the plasmas and to measure and predict their properties.

The other five articles have a narrower focus, each looking at a particular application: arc welding, plasma spraying in general and solution and suspension plasma spraying in particular, high-voltage circuit breakers and arcjet thrusters.

Arc welding and plasma spraying are examples of processes that are in widespread use across many industries. In arc welding, a direct-current transferred arc with a current of a few hundred amperes is used to melt and join metals. In plasma spraying, a direct-current non-transferred arc with a current of up to a thousand amperes is used to produce a highvelocity plasma jet, into which ceramic powders are injected; the powders are melted and blasted onto a substrate. Solution and suspension plasma spraying are relatively new variants under intensive development that allow deposition of nanostructured coatings.

High-voltage circuit breakers are essential components of the electrical distribution system; in this case, the goal is to extinguish the arc plasma as quickly as possible, so as to minimize the problems that can arise when a fault current, which can be up to several hundred kiloamperes, occurs in a circuit. Arcjet thrusters are used for propulsion in outer space; they use very much lower currents, typically just ten amperes, in a confined space to produce a supersonic plasma jet.

These are just a few examples of the many applications of thermal plasmas; nevertheless they give a picture of the diversity of applications of thermal plasmas. Taken as a whole, the articles provide an indication of the rich variety of problems that are available to researchers in the field.

The special issue is dedicated to the memory of Professor Joachim Heberlein, who died in February 2014. Professor Heberlein was a member of the Editorial Board of Plasma Chemistry and Plasma Processing, and one of the founders of the International Thermal Plasma Roundtable series of conferences that bring experts from academic and industrial research together to discuss issues such as those canvassed in this special issue. Jockel, as he was known by his friends and colleagues, was a greatly-respected and much-loved champion of thermal plasma research, and is sorely missed by the thermal plasma community. The special issue opens with an Obituary for Professor Heberlein. 